\title{
A unified approach to network location problems
}

\author{
Stefan Nickel *\& Justo Puerto ${ }^{\dagger}$
}

\begin{abstract}
In this paper we introduce a new type of single facility location problems on networks which includes as special cases most of the classical criteria in the literature. Structural results as well as a finite dominating set for the optimal locations are developed. Also the extension to the multi-facility case is discussed.
\end{abstract}

Keywords: Location, networks.

\section{Introduction}

One of the most important and well-developed branches in location theory is location problems on networks. Numerous surveys and textbooks (see [3], [12], [13], [11] and references therein) give evidence for this fact. The starting point of this development might be considered the node-dominance result of Hakimi [4] which we will show to be essential in this paper. In the existing literature mainly three types of objective functions are used: the median objective (sum objective), the centre objective (max objective) and the cent-dian objective (a convex combination of sum and max) (see [8] and the references therein for a description of these and many other facility location problems).

In this paper we will introduce a new type of objective function which will be shown to be a generalization of the most popular objective functions (mentioned above). The advantage of this new objective function will be the possibility of reproving a lot of

\footnotetext{
${ }^{*}$ Fachbereich Mathematik. Universität Kaiserslautern. Germany

${ }^{\dagger}$ Departamento de Estadisitca e Investigación Operativa. Universidad de Sevilla. Spain
} 
known results in a much easier way and getting more insight in the geometrical structure of the network with respect to different criteria.

More formally, let $\mathcal{N}=(\mathcal{G}, l)$ denote a network with underlying graph $\mathcal{G}=(\mathcal{V}, \mathcal{E})$, where the node set $\mathcal{V}=\left\{v_{1}, \ldots, v_{M}\right\}$ and the edge set $\mathcal{E}=\left\{e_{1}, \ldots, e_{N}\right\}$. We restrict ourselves to undirected graphs. Therefore, we write every edge $e \in \mathcal{E}$ as $\left[v_{i}, v_{j}\right]=$ $\left[v_{j}, v_{i}\right], v_{i}, v_{j} \in \mathcal{V}$.

Each edge $e \in \mathcal{E}$ has associated a positive length by means of the function $l: \mathcal{E} \rightarrow$ $\mathbb{R}_{+}$. By $d\left(v_{i}, v_{j}\right)$, we denote the length of the shortest path between $v_{i}$ and $v_{j}$ measured by $l$. Through $w: \mathcal{V} \rightarrow \mathbb{R}_{+_{0}}$, every vertex is assigned a non negative weight.

A point $x$ on an edge $e=\left[v_{i}, v_{j}\right]$ is defined as a pair $x=(e, t), t \in[0,1]$, with

$$
d\left(v_{k}, x\right):=d\left(x, v_{k}\right):=\min \left\{d\left(v_{k}, v_{i}\right)+t l(e), d\left(v_{k}, v_{j}\right)+(1-t) l(e)\right\} .
$$

The set of all the points of a network $(\mathcal{G}, l)$ is denoted by $\mathcal{P}(\mathcal{G})$. It should be noted that this set also contains the nodes $\mathcal{V}$.

Let

$$
d(x):=\left(w_{1} d\left(v_{1}, x\right), \ldots, w_{M} d\left(v_{M}, x\right)\right)
$$

and

$$
d_{\leq}(x):=\left(w_{(1)} d\left(v_{(1)}, x\right), \ldots, w_{(M)} d\left(v_{(M)}, x\right)\right)
$$

a permutation of the elements of $d(x)$ verifying

$$
w_{(1)} d\left(v_{(1)}, x\right) \leq w_{(2)} d\left(v_{(2)}, x\right) \leq \ldots \leq w_{(M)} d\left(v_{(M)}, x\right)
$$

For the sake of simplicity, let $d_{(i)}(x):=w_{(i)} d\left(v_{(i)}, x\right)$.

The ordered median problem on $\mathcal{N}$ is defined as

$$
\min _{x \in \mathcal{P}(\mathcal{G})} M_{\lambda}(x):=\sum_{i \in \mathcal{M}} \lambda_{i} d_{(i)}(x) \quad \text { with } \quad \lambda=\left(\lambda_{1}, \ldots, \lambda_{M}\right) \in \mathbb{R}_{+_{0}}^{M}
$$

where $\mathcal{M}:=\{1, \ldots, M\}$.

The function $M_{\lambda}(x)$ is called from now on the ordered median function. Note that the ordered median function is defined point-wise.

In this paper we consider the problem of finding a point $x \in \mathcal{P}(\mathcal{G})$ minimizing the ordered median function $M_{\lambda}$. According to the classification scheme introduced in [5] this problem will be referred to in the following as $1 / \mathcal{G} / \bullet / d(\mathcal{V}, \mathcal{G}) / \sum_{\text {ord }}$.

The reader may note that $1 / \mathcal{G} / \bullet / d(\mathcal{V}, \mathcal{G}) / \sum_{\text {ord }}$ is somehow similar to the wellknown median problem $\left(1 / \mathcal{G} / \bullet / d(\mathcal{V}, \mathcal{G}) / \sum\right)$. We will show in this paper that $1 / \mathcal{G} / \bullet$ $/ d(\mathcal{V}, \mathcal{G}) / \sum$ as well as other classical network location problems are special cases of $1 / \mathcal{G} / \bullet / d(\mathcal{V}, \mathcal{G}) / \sum_{\text {ord }}$ 
The rest of the paper is organized as follows. In the next section we show some fundamental properties of $1 / \mathcal{G} / \bullet / d(\mathcal{V}, \mathcal{G}) / \sum_{\text {ord }}$. After that the extension to the multifacility case $\left(N / \mathcal{G} / \bullet / d(\mathcal{V}, \mathcal{G}) / \sum_{\text {ord }}\right)$ is discussed. The paper ends with some conclusions and an outlook to further research.

\section{Properties of $1 / \mathcal{G} / \bullet / d(\mathcal{V}, \mathcal{G}) / \Sigma_{\text {ord }}$}

In this section we state the fundamental properties of the problem $1 / \mathcal{G} / \bullet / d(\mathcal{V}, \mathcal{G}) / \sum_{\text {ord }}$. We will present a localization result which generalizes the well-known Hakimi theorem [4] and gives some insight in the connection between median and center problems. In addition, we show how most of the location problems previously considered on networks can be seen as particular instances of this new problem.

For all $v_{i}, v_{j} \in \mathcal{V}, i \neq j$ define

$$
E Q_{i j}:=\left\{x \in \mathcal{P}(\mathcal{G}): w_{i} d\left(v_{i}, x\right)=w_{j} d\left(v_{j}, x\right)\right\}
$$

and let $\mathcal{E Q}:=\bigcup_{\substack{i, j \\ i \neq j}} E Q_{i j}$.

It is well-known that the points in $\mathcal{E} \mathcal{Q}$ are the so called bottleneck points of $\mathcal{N}$ [8]. These points establish a partition on $\mathcal{N}$ with the property that for two consecutive elements $a, b \in \mathcal{E} \mathcal{Q}$ the permutation which gives the order to the vector $d_{\leq}(x)$ is the same for all $x \in[a, b]$. Here consecutive means, that there is no other $c \in \mathcal{E} \mathcal{Q}$ on the shortest path between $a$ and $b$.

Theorem 2.1 An optimal solution for $1 / \mathcal{G} / \bullet / d(\mathcal{V}, \mathcal{G}) / \sum_{\text {ord }}$ can always be found in the set $C$ and $:=\mathcal{E} \mathcal{Q} \cup \mathcal{V}$.

Proof. Starting from the original graph $\mathcal{G}$, build a set of new graphs $\mathcal{G}_{1}, \ldots, \mathcal{G}_{K}$ by inserting all points of $\mathcal{E} \mathcal{Q}$ as new nodes. Now every subgraph $\mathcal{G}_{i}$ is defined by either

1. Two consecutive elements of $\mathcal{E} \mathcal{Q}$ on an edge or

2. An element $v_{i} \in \mathcal{V}$ and the adjacent elements of $\mathcal{E} \mathcal{Q}$

and the corresponding edges. In this situation for every subgraph $\mathcal{G}_{i}$ the permutation of $d_{\leq}(x)$ is constant (by definition of $\mathcal{E} \mathcal{Q}$ ). Therefore for all $x \in \mathcal{P}\left(\mathcal{G}_{i}\right)$ we have

$$
\sum_{i \in \mathcal{M}} \lambda_{i} d_{(i)}(x)=\sum_{i \in \mathcal{M}} \lambda_{i} w_{\pi(i)} d\left(v_{\pi(i), x}, x\right)
$$


where $\pi \in \Pi(\mathcal{M})$, where $\Pi(\mathcal{M})$ is defined as the set of all permutations of $\mathcal{M}$. Therefore we can replace the objective by a classical median-objective. Now we can apply Hakimi's node dominance result in every $\mathcal{G}_{i}$ and the result follows.

Theorem 2.1 gives raise to some geometrical subdivision of the network $\mathcal{N}$. Like indicated in the proof of Theorem 2.1 we can assign every subgraph $\mathcal{G}_{i}, i=1, \ldots, k$ a M-tuple giving in the i-th position the i-th nearest vertex to all points in $\mathcal{G}_{i}$. As an example we have in Figure 1 a graph with 3 nodes and all weights $w_{i}$ and all lengths are 1.

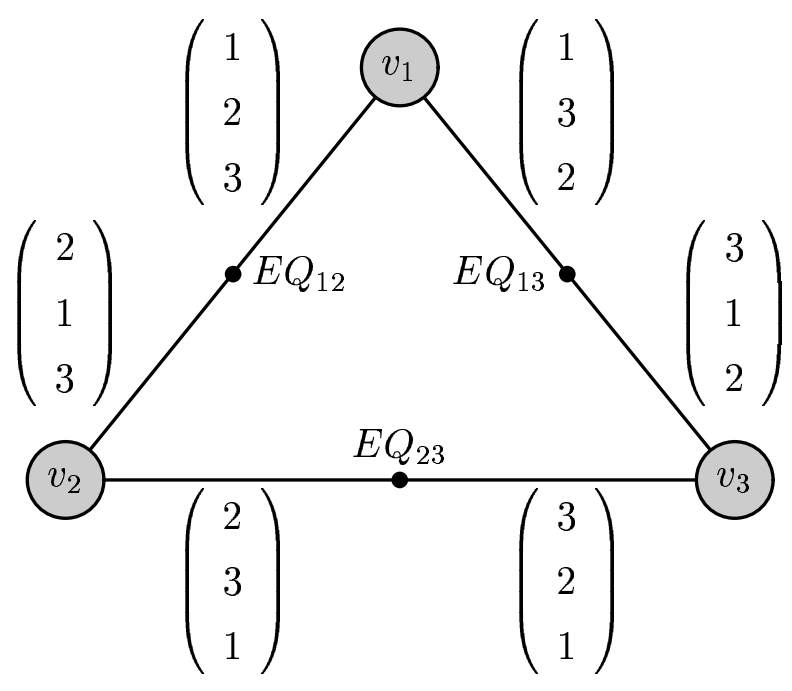

Figure 1: A 3-node network with bottleneckpoints and the geometrical subdivision.

This partition can be seen as a kind of higher order Voronoi diagram of $\mathcal{N}$ quite related to the Voronoi partition of networks introduced in[7].

We will now show on the basis of Theorem 2.1 that many well-known location problems on networks are particular instances of $1 / \mathcal{G} / \cdot / d(\mathcal{V}, \mathcal{G}) / \sum_{\text {ord }}$ for specific choices of the set of $\lambda$-weights.

\section{Theorem 2.2}

1. $1 / \mathcal{G} / \lambda_{m}=\frac{1}{M} / d(\mathcal{V}, \mathcal{G}) / \sum_{\text {ord }} \hat{=} 1 / \mathcal{G} / \frac{w_{m}}{M} / d(\mathcal{V}, \mathcal{G}) / \sum$.

2. $1 / \mathcal{G} / \lambda_{M}=1, \lambda_{m}=0, m \neq M / d(\mathcal{V}, \mathcal{G}) / \sum_{\text {ord }} \hat{=} 1 / \mathcal{G} / \bullet / d(\mathcal{V}, \mathcal{G}) / \max$.

3. $1 / \mathcal{G} / \lambda_{1, \ldots, M-1}=\frac{\hat{\lambda}}{M}, \lambda_{M}=\frac{M+\hat{\lambda}(1-M)}{M} / d(\mathcal{V}, \mathcal{G}) / \sum_{\text {ord }}$

$$
\hat{=} 1 / \mathcal{G} / \bullet / d(\mathcal{V}, \mathcal{G}) / \text { cent }- \text { dian }
$$

4. $1 / \mathcal{G} / \lambda_{1, \ldots, k-1}=0, \lambda_{k, \ldots, M}=\frac{1}{k} / d(\mathcal{V}, \mathcal{G}) / \sum_{\text {ord }} \hat{=} 1 / \mathcal{G} / \bullet / d(\mathcal{V}, \mathcal{G}) / k-$ centra 
Proof. Let $x \in \mathcal{P}(\mathcal{G})$ and let $\mathcal{G}_{1}, \ldots, \mathcal{G}_{K}$ be the subgraphs as defined in the proof of Theorem 2.1. Therefore $x \in \mathcal{P}\left(\mathcal{G}_{k}\right)$ for some $k \in\{1, \ldots, K\}$ and we can write

$$
M_{\lambda}(x)=\sum_{i \in \mathcal{M}} \frac{1}{M} d_{(i)}(x)=\sum_{i \in \mathcal{M}} \frac{1}{M} w_{\pi(i)} d\left(v_{\pi(i)}, x\right)=\frac{1}{M} \sum_{i \in \mathcal{M}} w_{i} d\left(v_{i}, x\right)
$$

with $\pi \in \Pi(\mathcal{M})$. Since the value of the sum does not depend on the permutation $\pi$ we can choose $\pi=i d$ and get the classical median function, which concludes the proof for Part 1.

Starting with the $\lambda$-weights of Part 2 we get

$$
M_{\lambda}(x)=\sum_{i \in \mathcal{M}} \lambda_{i} d_{(i)}(x)=\lambda_{M} d_{(M)}(x)=\max _{i \in \mathcal{M}} w_{i} d\left(v_{i}, x\right)
$$

and Part 2 is shown.

The proofs for the remaining two parts are analogous to the parts shown and are therefore omitted here.

Lots of known results follow directly from Theorem 2.2. Although this formulation has been related to some well-known models, it is also possible to formulate many other models which have not been addressed yet. An example for a problem which is not addressed yet is the following

$$
\min \sum_{i=1}^{M} \lambda_{i} d_{(i)}(v) \quad \text { with } \lambda_{i}=1, i \leq k, \lambda_{i}=0, i>k
$$

which is the contrary to the k-centra problem introduced by Andreatta and Mason [1] because the distance to the nearest $k$ facilities is minimized.

For algorithmic purposes one should note that the set $\mathcal{E} \mathcal{Q}$ coincides with the set $L M_{i j}$ introduced by Kariv and Hakimi [9]. In that paper they prove that their algorithm (which is shown to be optimal) determines $\mathcal{E} \mathcal{Q}$ in $\mathcal{O}(|\mathcal{V}||\mathcal{E}| \log (|\mathcal{V}|))$ time.

\section{Extension to $N / \mathcal{G} / \bullet / d(\mathcal{V}, \mathcal{G}) / \Sigma_{\text {ord }}$}

In this section we deal with the multi-facility extension of the ordered median problem. In the previous section we proved that the set $\mathcal{V} \cup \mathcal{E} \mathcal{Q}$ always contains the set of optimal solution of the problem. It might seem natural to expect that the same result holds for the $N$-facility case as it happens for the $N$-center problem. However, the following example taken from Perez-Brito et al. [2] shows that this property fails to be true.

Counterexample. Consider the tree network drawn in Figure 2 


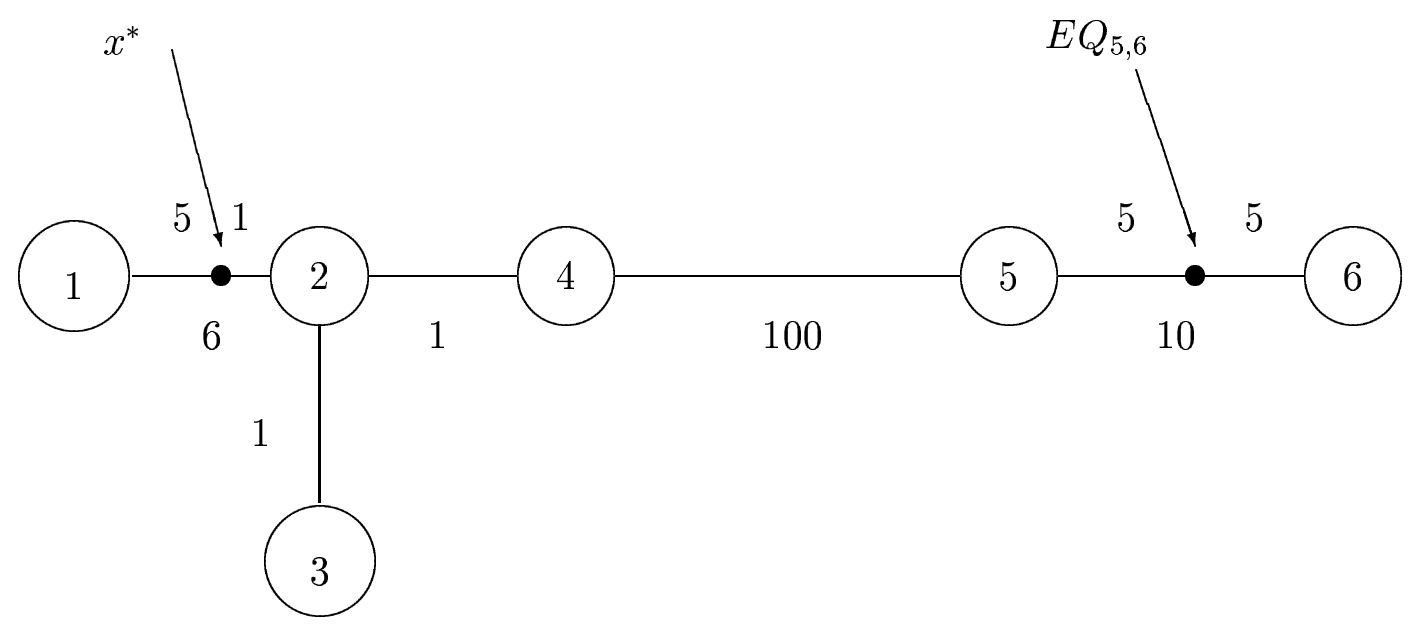

Figure 2: Counterexample

We want to consider the $2-\lambda$ cent-dian with $\lambda=0.8$. All the weights are equal to 1. It is very easy to see that two clusters of nodes appear; the first one with vertices $1,2,3,4$ and the second one with vertices 5 and 6 . The best solution considering the different local centers and vertices combinations is given by $X=\left\{E Q_{1,4}, E Q_{5,6}\right\}$ with objective value of 8.6. However, if we consider the point $x^{*}$ at distance 5 from $v_{1}$ which is neither a vertex nor an EQ point, the set $X^{*}=\left\{x^{*}, E Q_{5,6}\right\}$ has as objective value 8.0 .

The rest of this section is devoted to deal with the $N$-facility weighted ordered median problem $N / \mathcal{G} / \bullet / d(\mathcal{V}, \mathcal{G}) / \sum_{\text {ord }} . \quad N / \mathcal{G} / \bullet / d(\mathcal{V}, \mathcal{G}) / \sum_{\text {ord }}$ is a problem which consists of finding a set $X_{N}=\left\{x_{1}, \ldots, x_{N}\right\}$ that minimizes the following objective function

$$
\min _{X_{N}} \sum_{i=1}^{M} \lambda_{i} d_{(i)}\left(X_{N}\right)
$$

where we impose additionally that $\lambda_{1} \geq \lambda_{2} \geq \ldots \geq \lambda_{M}$.

The main result of this section establishes a theorem similar to the well-known Hakimi's theorem which states that always exists an optimal solution $X_{p}^{*} \subseteq \mathcal{V}$.

Theorem 3.1 $N / \mathcal{G} / \lambda_{1} \geq \lambda_{2} \geq \ldots \lambda_{M} / d(\mathcal{V}, \mathcal{G}) / \sum_{\text {ord }}$ has always an optimal solution $X_{N}^{*}$ contained in $\mathcal{V}$.

Proof. Since by hypothesis $\lambda_{1} \geq \lambda_{2} \geq \ldots \geq \lambda_{M}$ we have that

$$
M_{\lambda}\left(X_{N}\right)=\sum_{i=1}^{M} \lambda_{i} d_{(i)}\left(X_{N}\right)=\min _{\pi \in \Pi(\mathcal{M})}\left\{\sum_{i=1}^{M} \lambda_{i} d_{\pi(i)}\left(X_{N}\right)\right\} .
$$


Assume that $X_{N} \not \subset \mathcal{V}$.

Then there must exist $x_{i} \in X_{N}$ with $x_{i} \notin \mathcal{V}$. Let $e=[v, w]$ the edge containing $x_{i}$ and $l(e)$ its length. Denote by $X_{N}(s)=X_{N} \backslash\left\{x_{i}\right\} \cup\{x(s)\}$ where $x(s)$ is the point on $e$ with $d(v, x(s))=s, s \in[0, l(e)]$.

The function $g$ defined as $g(s)=\sum_{i=1}^{M} \lambda_{i} d_{(i)}\left(X_{N}(s)\right)$ is concave for all $s \in[0, l(v)]$ because it is the composition of a concave and a linear function, i.e.

$$
g(s)=\min _{\pi \in \Pi(\mathcal{M})}\left\{\sum \lambda_{i} d_{\pi(i)}\left(X_{N}(s)\right)\right\}
$$

and each

$d_{\pi(j)}\left(X_{N}(s)\right)=\min \left\{d\left(v_{\pi(j)}, x_{1}\right), \ldots, \min \left\{d\left(v_{\pi(j)}, a\right)+s, d\left(v_{\pi(j)}, b\right)+l(v)-s\right\}, \ldots, d\left(v_{\pi(j)}, x_{N}\right)\right\}$

is concave.

Hence, $g(s)=F\left(X_{N}(s)\right) \geq \min \left\{F\left(X_{N}(0)\right), F\left(X_{N}(l(v))\right\}\right.$ and the new solution set $X_{N}(s)$ contains instead of $x_{i}$ one vertex of $\mathcal{V}$.

Repeating this scheme a finite number of times the result follows.

Although this result is new similarities can be found with the correction given by Hooker et al. [8] to the dominance result (see Result 7 in [8]) of Weaver and Church [14] for the so called vector assignment N-median model.

\section{Conclusions}

In this paper we have introduced a new class of network location problems which we have shown to contain many of the classical problems as special cases. This approach helps to get more insight in the differences and the common properties of center and median location problems.

A classification scheme has been used for simplifying the understanding of the relation between the different classes of problems. Also a lot of new problems can be modeled and solved in polynomial time using this approach.

Finally the frontiers of this approach are shown by a counterexample for the multifacility case. Therefore the future research will include an appropriate extension to multi-facility problems. Besides this, also multi-criteria ordered Weber problems are under research. 


\section{Acknowledgements}

The research of the first author has been partially supported by Spanish DGICYT grant n. PB93-0927. The research of the second author is partially supported by a grant of the Deutsche Forschungsgemeinschaft.

\section{References}

[1] Andreatta G. \& Mason F.M. (1985) "Properties of the k-Centra in a tree network", Networks, 15, 21-25.

[2] Perez-Brito D., Moreno-Pérez, J. \& Rodríguez-Marín, I. (1996) "Finite dominating set for the p-facility cent-dian network location problem", Working paper of Universidad de La Laguna.

[3] Drezner Z. (EDitor) (1995) "Facility Location. A survey of applications and methods" Springer New York.

[4] Hakimi S.L. (1964) "Optimum location of switching centers and the absolute centers and medians of a graph", Operations Research, 12, 450-459.

[5] Hamacher H. \& Nickel S. (1993) "Multicriteria planar location problems", Technical Report 227, accepted by EJOR.

[6] Hansen P., Labbé M. \& Thisse J.F. (1991) "From the median to the generalized center", R.A.I.R.O. O.R., 25, n. 1, 73-86.

[7] Hakimi S.L., Labbé M. \& Schmeichel E. (1992) "The Voronoi partition of a network and its applications in location theory", ORSA Journal of Computing, 4, n. $4,412-417$.

[8] Hooker J.N., Garfinkel R.S. \& Chen C.K. (1991) "Finite dominating sets for network location problems", Operations Research, 39, n. 1, 100-118.

[9] Kariv O. \& HaKimi S.L. (1979) "An algorithmic approach to network location problems I: The p-centers", SIAM Journal of applied mathematics, 37, 513-538.

[10] Kariv O. \& HaKimi S.L. (1979) "An algorithmic approach to network location problems II: The p-medians", SIAM Journal of applied mathematics, 37, 539-560. 
[11] Labbé M., Peeters D. \& Thisse J.-F. (1995) "Location on Networks" , Handbooks in OR \& $M S$, edited by M. O. Ball et al. Vol. 8, Elsevier

[12] Mirchandani P.B. \& Francis R.L. (Editors) (1990) "Discrete Location Theory" John Wiley and Sons New York.

[13] Puerto J. (Editor) "Lecturas en Teoria de Localizacion" , Secretariado de Publicaciones de la Universidad de Sevilla. Serie Ciencias n.46. (in Spanish).

[14] Weaver J.R. \& Church R.L. (1985) "A median location model with nonclosets facility service", Transportation Science, 7, 18-23. 